Abstract

AGU 2000 Fall Meeting

H09 Quantifying Predictive Uncertainty of Hydrogeologic Flow and Transport Models

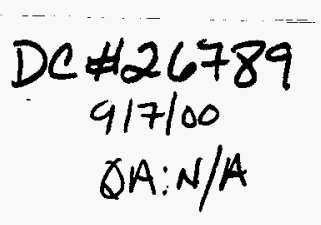

Conveners: S.P. Neuman and M.C. Hill

\title{
Impact of Parameter Uncertainty, Variability, and Conceptual Model Errors on Predictions of Flow Through Fractured Porous Media
}

\author{
S. Finsterle \\ Lawrence Berkeley National Laboratory \\ 1 Cyclotron Road, MS 90-1116 \\ Berkeley, CA 94720 \\ SAFinsterle@lbl.gov \\ 1-510-486-5205 \\ $1-510-486-5686$
}

Model predictions are affected by uncertainty in input parameters, stochastic variability in formation properties, computational roundoff and cancellation errors, and errors in the conceptual model. The source, nature, and relative magnitude of these errors vary considerably, depending on the physical processes involved, the quality and amount of available characterization data, and the overall objective of the study. We examined various types of uncertainties and their propagation with a predictive model that simulates a water pulse flowing through an unsaturated, fractured porous medium. The propagation of the water pulse depends not only on the hydraulic properties of the fracture network, but also on the strength of fracture-matrix interactions and the storage capacity of the matrix. Different predicted variables (such as local saturation changes, total amount of water retarded in the matrix, or first arrival of water at a certain depth) depend on different parameters and thus show different uncertainty structures. The strong nonlinearities inherent in such a system require the use of Monte Carlo simulations. These simulations investigate the spread of model predictions as a result of changes in spatial variability and uncertainty in key input parameters. We also discuss the role of conceptual-model formulation and parameter estimation in the development of reliable prediction models. We observe that systematic errors in the conceptual model often render probabilistic uncertainty analyses meaningless if not misleading. Nevertheless, sensitivity analyses provide useful insight into the system behavior and help design experiments that eventually would reduce prediction uncertainties.

This work was supported by the U.S. Department of Energy under Contract No. DEAC03-76SF00098. 